\title{
Plant hormone receptors: perception is everything
}

\author{
Brenda Chow ${ }^{1}$ and Peter McCourt ${ }^{1,2}$ \\ ${ }^{1}$ Department of Botany, University of Toronto, Toronto M5S 3B2, Canada
}

\begin{abstract}
Despite the bewildering ability of higher plants to change their development with respect to the environment, there appear to be only a few hormones that function to organize growth and development. With the recent identification of three plant hormone receptors, the molecular identities of all the major plant receptors are now known. Some plant hormones such as cytokinins, ethylene, and brassinosteroids (BR) use well-characterized signaling modules such as those involving receptor kinases, but in the case of the ethylene and BR receptors, there appear to be additional functions aside from the hormone they perceive. Auxin and gibberellin perception require unique mechanisms where the receptors are components involved in ubiquitination-dependent proteolysis. With plant hormone receptors in hand, comparisons can now be made between plants and other kingdoms as to how hormones control growth and development.
\end{abstract}

Multicellular organisms, whether plants or animals, coordinate their growth and development at a tissue and organ level by using extracellular signaling molecules to communicate between cells. In animals, these extracellular signals usually take the form of small proteins or peptides, like insulin, or small organic-based hormone molecules, such as steroids or retinoids. In the case of plants, the organic class of hormones has historically dominated since these molecules influence numerous aspects of growth and development (Davies 1995). Intriguingly, although a number of the plant hormone structures have similar animal counterparts, plant hormones seem to have a much broader range of function (Fig. 1). A single plant cell can respond to more than one hormone, while a single hormone can affect different tissues in different ways. Obviously, any understanding of how this developmental complexity is initiated by these simple organic molecules first requires an understanding of how they are perceived. Until recently, however, the molecular properties of plant hormone perception were fragmentary compared with animal studies

[Keywords: Arabidopsis; hormones; development; signal transduction] ${ }^{2}$ Corresponding author.

E-MAIL mccourt@botany.utoronto.ca; FAX (416) 978-5878.

Article is online at http://www.genesdev.org/cgi/doi/10.1101/gad.1432806. due to the difficulties in biochemical purification of receptors.

Unlike animal systems, where organs and glands are discrete, plant development is a continuum of growth and differentiation in which cells and tissues both produce and respond to many hormones. Biochemical analysis is further compromised by the cell wall and the plethora of secondary chemical products that plants synthesize. Fortunately, with the arrival of modern Arabidopsis genetics, mutations were identified that increased or decreased the response of a whole plant to a particular hormone. With the mutants in hand, genes could be isolated without the need for sophisticated biochemistry, and in the past few years, receptors to many of the major plant hormones have been identified. This has allowed biochemical studies and has also initiated a comprehensive comparison of how multicellular plants and animals, which most likely evolved independently, use similar organic-based hormones to organize development.

In this review, because of the strong influence of genetic analysis on plant hormone studies, we have limited ourselves to receptors where in vivo studies have provided the reagents for in vitro experiments. We have focused on five receptors, as a sufficient body of work exists for these to allow both informed mechanistic conclusions and general comparisons to animal counterparts. We have not dealt with the recent identification of a receptor for abscisic acid (ABA) as the role of this receptor in general ABA signaling is not clear at this time (Razem et al. 2006). We have also not dwelt on the subtleties of mutant screens or details of phenotypes as these topics have been covered in detail by a large number of excellent reviews. Sufficient information is now available to allow receptors to be classified with respect to their biochemical properties rather than by the hormone they perceive. This type of organization allows comparisons not only between different plant hormone systems but also, where possible, to receptors in other kingdoms.

\section{Two-component-based hormone receptors}

Two-component regulators derive their name from the transfer of phosphates between a collection of signature 


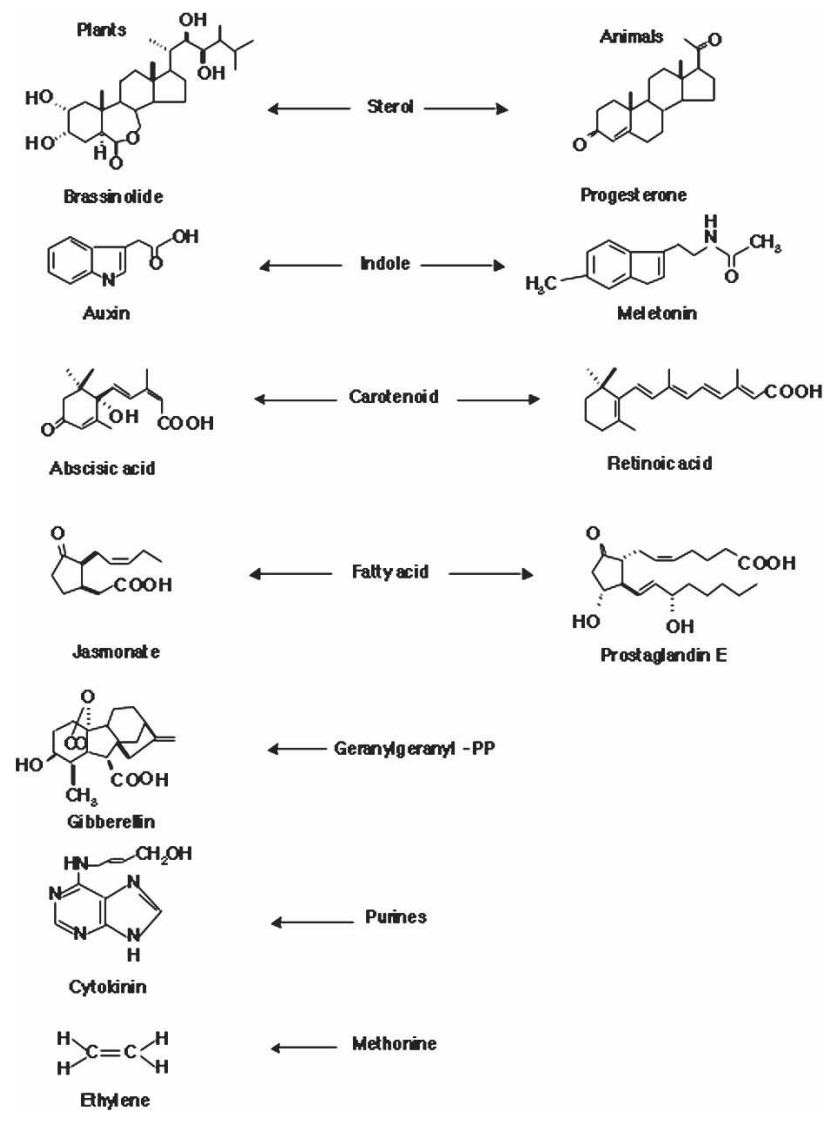

Figure 1. Chemical structures of different organic-based hormones found in plants and animals. The names in the center represent the precursors from which the hormones are derived.

proteins. This starts when the first component perceives an input signal to cause an autophosphorylation of a conserved histidine in the transmitter domain (Fig. 2A). The signal is then relayed through a phosphotransfer to a conserved aspartate residue in the receiver domain of the second component, often called the response regulator. Two-component regulators were first defined in prokaryotes, where they are quite prevalent, often representing up to $1 \%$ of the genome (Mizuno 1997). To date, no twocomponent regulators have been found in animals; however, they have been identified in both fungi and plants. The fungal and plant two-component systems are more complicated than the canonical prokaryotic system in that the input histidine kinase domain itself often carries a receiver domain (Fig. 2B). Moreover, an intermediate, designated HPt for histidine phosphotransfer protein can also participate in the phosphate transfer between the histidine kinase and the receiver domain. Three-dimensional protein studies show that HPt domains share similarity with parts of the transmitter domain (Kato et al. 1997).

In Arabidopsis, eight two-component input regulators genes have been functionally identified as hormone receptors (Fig. 3). Three are involved in cytokinin perception, and five encode ethylene receptors. The first cytokinin receptor was identified by taking advantage of the ability of exogenous cytokinins to cause greening in Arabidopsis plant tissue culture. An insensitive mutant was isolated, which led to the identification of the membrane-bound two-component input regulator, CRE1 (Inoue et al. 2001). Subsequently, two close homologs, AHK2 and AHK3, were also identified (Suzuki et al. 2001; Yamada et al. 2001; Higuchi et al. 2004). All three gene products have been shown to bind cytokinin and complement yeast and bacterial two-component mutants in a cytokinin-dependent manner (Fig. 3A). In Arabidopsis, there are five genes that encode HPt proteins, designated AHPs, and it appears that these intermediates transmit the membrane perceived cytokinin signal to the response regulators (ARRs) located in the nucleus (Hwang and Sheen 2001).

Because a small gene family defines the cytokinin receptors, it was perhaps not surprising that single loss-offunction mutations were not lethal and, for the most part, allowed plants to grow normally (Higuchi et al. 2004). Combinations of double and triple mutations did expose abnormal developmental phenotypes, but surprisingly, embryogenesis and early seedling patterning were relatively normal. Although it is possible that other cytokinin perception systems exist, these results hint that the traditional view of an essential role for cytokinins in plant growth and development may be exaggerated.

The first genes encoding ethylene receptors were also identified through genetic screens for mutants that did not respond to hormone application. These studies led to the classification of five genes (ETR1, ETR2, ERS1, ERS2, EIN4) that share sequence similarity with those enocoding the two-component input regulators (Bleecker et al. 1988; Chang et al. 1993; Hua et al. 1995; Hua and Meyerowitz 1998; Sakai et al. 1998). Heterologous expression studies in yeast have clearly defined the signal input domain that perceives ethylene as a hydrophobic region at the $\mathrm{N}$ terminus of members of the ethylene receptor family (Chen et al. 2005; O'Malley et al. 2005). However, unlike the cytokinin story, the transmitter domain has been more difficult to understand for a variety of reasons. First, although truncation of the histidine kinase domain results in the loss of signal output, site-directed mutagenesis that specifically eliminates kinase activity only has modest effects on ethylene signaling $(\mathrm{Qu}$ and Schaller 2004). Second, phylogenetic analysis can divide the ethylene receptors into two subfamilies based on the presence or absence of conserved subdomains characteristic for histidine kinases (Fig. 3B). Finally, some receptor family members contain a C-terminal receiver domain that may be phosphorylated by the histidine kinase domain, but the presence of a receiver domain does not correlate with the presence of a histidine kinase domain. However, since gain-of-function mutations in any of the receptors confer insensitivity to ethylene, it appears that all five genes can contribute to ethylene signaling. The dominant nature of these mutations suggests that mutant receptors may interact with wild-type receptors to transduce the ethylene signal.

As with the cytokinin receptors, individual loss-of- 

system. (A) The two-step system found only in prokaryotes. The input domain binds a signal molecule, resulting in the phosphorylation (green-encircled P) of a conserved histidine in the transmitter domain. This phosphoryl group is subsequently transferred to the receiver domain on the response regulator. As a result, the output domain is activated to transduce the signal. (B) The multistep derivation of the two-component system found in prokaryotes and some eukaryotes. Overall phosphotransfer is similar to $A$ with the exception of intermediate components acting between the transmitter and output domains. These include receiver domains fused to the transmitter domain and a separate intermediate $\mathrm{Hpt}$ domain that can receive a phosphoryl group from a receiver domain. In all cases, phosphotransfers can be bidirectional. H (histidine), $\mathrm{N}$ (asparagine), G (glycine), and $\mathrm{F}$ (phenylalanine) represent conserved blocks characteristic of functional histidine kinases.
Figure 2. The prototypical two-component
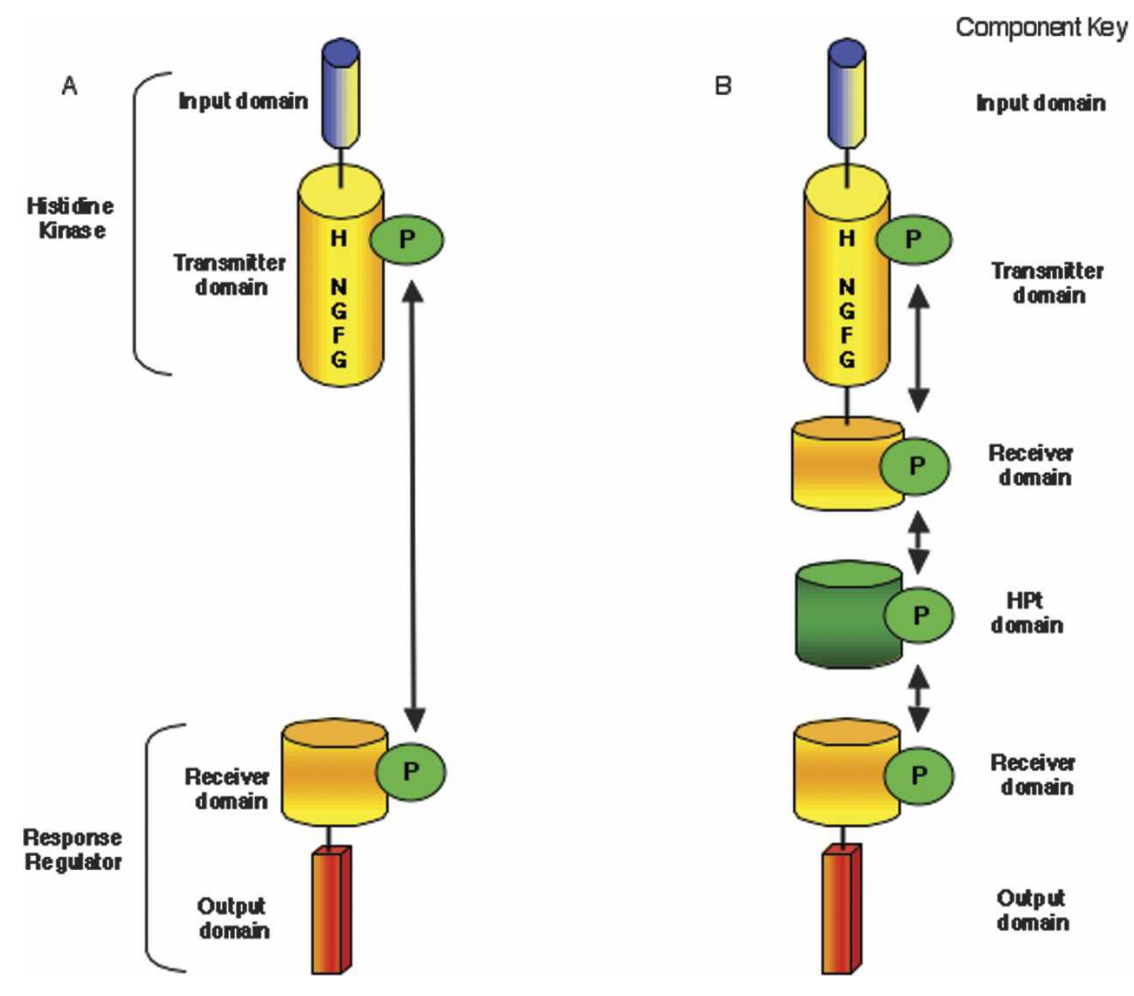

function mutations in the ethylene receptor genes caused little change in plant growth while multiple combinations resulted in developmental phenotypes (Hua and Meyerowitz 1998). However, unlike the cytokinin response where loss of more receptors decreased cytokinin responsiveness, decreasing the number of functional ethylene receptors resulted in plants with a constitutive ethylene response (Hua and Meyerowitz 1998). The most parsimonious explanation for this result is that the family of ethylene receptors normally functions in the absence of ethylene and that addition of the gas inhibits this function (Fig. 3B). This model fits well with the immediate downstream component of the receptors, CTR1, since this serine/threonine protein kinase is a negative regulator of the ethylene response in Arabidopsis (Kieber et al. 1993). In the absence of ethylene, the receptors activate CTR1, which in turn suppresses the ethylene response (Fig. 3B). When ethylene binds the receptors, CTR1 activation is inhibited, releasing downstream components to activate the ethylene pathway. Protein interaction studies and colocalization of both ETR1 and CTR1 protein to the endoplasmic reticulum (ER) support the genetic interactions (Clark et al. 1998; Cancel and Larsen 2002; Chen et al. 2002; Gao et al. 2003). Because specific mutations in CTR1 that disrupt ETR1-CTR1 interactions cause movement of CTR1 to the cytosol and genetic removal of two or more ethylene receptors also results in CTR1 protein redistribution, it appears that the ethylene receptors act as a scaffolding protein for CTR1 at the ER (Gao et al. 2003; Huang et al. 2003). Therefore, ethylene receptors are quite unlike cytokinin or other two-component receptors in that there is no phospho-relay to a typical HPt domain protein or a response regulator.

The oddities of the ethylene "two-component" receptors also apply to the way ethylene itself interacts with the receptor. Like other hormone ligands, ethylene is active at nanomolar concentrations. However, unlike most hormones, which are generally complex with respect to molecular shape, ethylene simply consists of two carbons. The lack of multiple contact points between ethylene and its receptor would not allow for the many weak interactions that are usually required to achieve high-affinity ligand-receptor specificity. This peculiarity was explained by the demonstration that a copper ion associates with the ethylene receptor and is required for binding of ethylene in both Arabidopsis and heterologous yeast systems expressing ETR1 (Schaller and Bleecker 1995; Rodriguez et al. 1999). Interestingly, the cyanobacterium Synechocystis strain 6803 contains an ethylene receptor ortholog, which also requires a copper cofactor to bind ethylene at a high affinity (Rodriguez et al. 1999). Although Synechocystis does not synthesize or respond to ethylene, the conservation between bacterial and higher plant genes suggests that the progenitor to the ethylene receptor in plants was co-opted from a cyanobacterial ancestor after plant cells acquired the chloroplast during endosymbiosis.

This model implies that the progenitor ethylene receptor may have evolved to influence an already existing plant signaling pathway. Models of signaling pathway evolution have suggested that these pathways evolve in reverse order from the target transcription factor to the first step, which is the receptor and ligand combination 


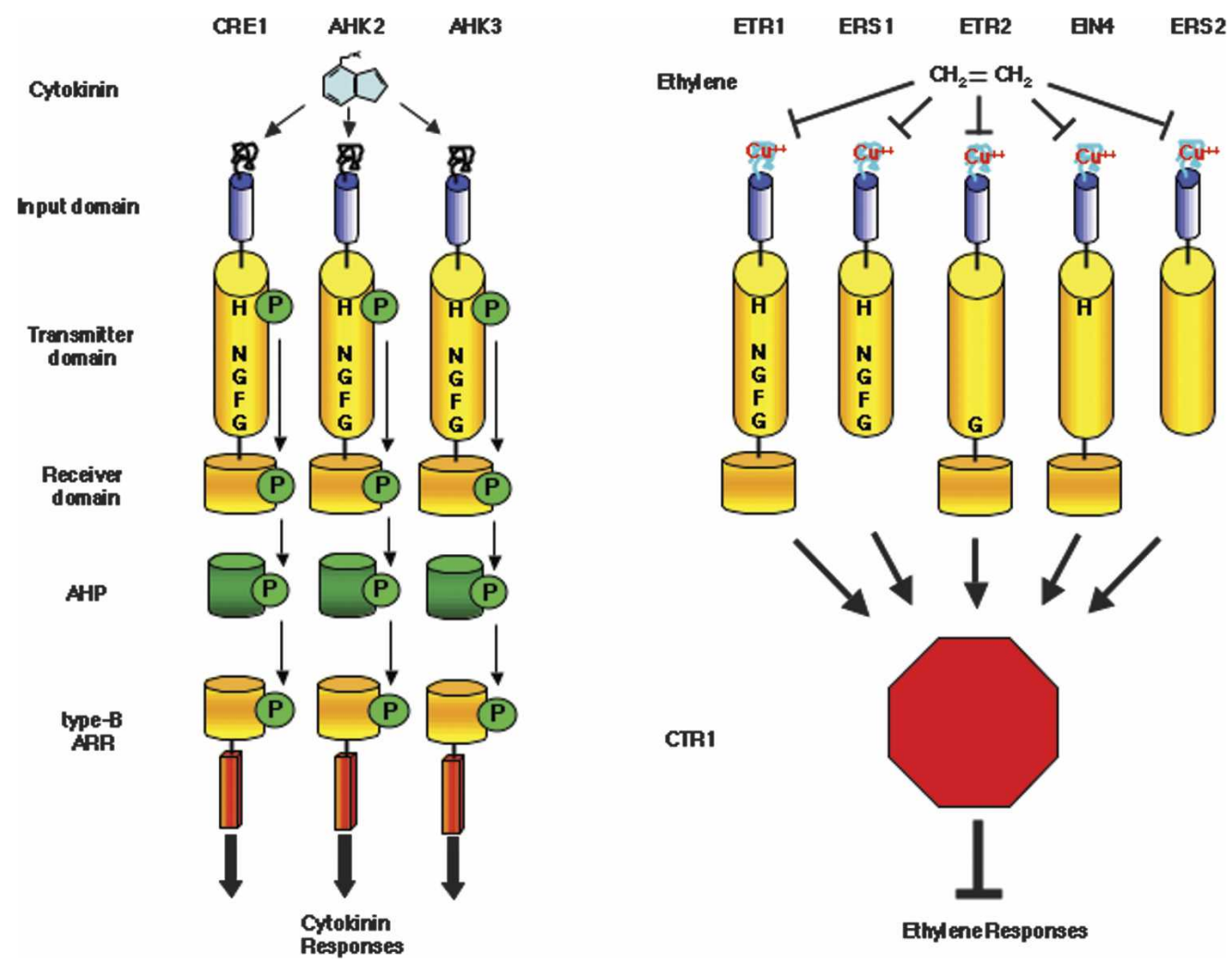

Figure 3. Structural relationships within and between two-component regulators involved in cytokinin and ethylene perception. $(A)$ In Arabidopsis, three cytokinin receptors (CRE1, AHR2, and AHR3) all bind cytokinins at their extracellular input domain. This results in a phospho-relay that eventually activates cytokinin responses. AHP and the type-B ARR are the Arabidopsis equivalents of the Hpt domain and response regulator proteins, respectively. (B) Five ethylene receptors (ETR1, ERS1, ETR2, EIN4, and ERS2) exist in Arabidopsis that all bind ethylene in their extracellular domain. Various forms of the transmitter domain exist on each receptor so, in principle, not all receptors can transfer phosphate groups. However, genetic analysis suggests that all of the ethylene receptors activate their downstream target component (CTR1) in the absence of the gas, and that binding of ethylene to the receptors inhibits this activation of CTR1.

(Wilkins 1995). Often new components act as neomorphic and dominant-negative units that reverse the function of the previous step. This would appear to hold true for the upper part of the ethylene signaling pathway in which CTR1 acts negatively on EIN2, ethylene receptors act positively on CTR1, and, finally, ethylene acts negatively on the receptors. The idea that the ethylene receptor was a late addition to a pre-existing pathway may explain the major paradox in ethylene signaling regarding how enhanced sensitivity can occur with a reduction in receptor number (Cancel and Larsen 2002). The observation that glucose can enhance the degradation of EIN3, a key transcriptional regulator at the bottom of the ethylene response pathway, hints that the ethylene pathway has other modulators (Yanagisawa et al. 2003). If the role of the bottom of the ethylene response pathway is to modulate sugar responses, which in turn determines cell growth and expansion, then the role of ethylene and its upstream receptor may simply be to inhibit a carbon response pathway.

\section{Leucine-rich repeat (LRR)-based hormone receptors}

In both plants and animals, steroid-based molecules have been identified that act as hormones for directing growth and development. In plants, the best described steroid hormone is brassinosteroid (BR). The first interest in BR arose from its growth promoting qualities upon application. The isolation of Arabidopsis mutants deficient in BR biosynthesis clearly demonstrated that BR is in fact a general regulator of plant growth and development (Li et al. 1996; Szekeres et al. 1996). The identification of mutations affecting BR perception involved screening for Arabidopsis mutants that showed normal root growth on exogenous BR or by screening for dwarf mutants that mimicked BR auxotrophic phenotypes that were not rescued by exogenous BR (Clouse et al. 1996; Li and Chory 1997; Noguchi et al. 1999).

In both cases, these approaches uncovered many alleles of a single locus, designated BRI1, which encodes a plasma membrane-associated LRR receptor-like kinase (LRR-RLK) (Li and Chory 1997). The LRR is a common protein motif of usually 20-29 residues that is found in a number of proteins with diverse functions throughout all kingdoms (Fig. 4A; Kobe and Kajava 2001). Plant receptors have an extracellular LRR domain that is fused to an intracellular serine/threonine kinase domain. In animals, LRR-based receptors transduce their signals through intracellular protein-protein interactions rather 
Figure 4. LRR receptors. (A) Comparison of four types of LRR receptors found in plants and animals: BRI1, an Arabidopsis $\mathrm{BR}$ receptor; CLV1, a receptor involved in binding the small peptide CLV3 that downregulates meristem size in Arabidopsis; Cf9 , a receptor involved in plant pathogen responses in tomato; and Toll, an embryo patterning and innate immunity receptor in Drosophila. (B) Mechanism of BR perception. Normally the BRI1 and BAK1 LRR kinases are inactive homodimers. Upon BR binding, the BRI1 and BAK1 proteins aggregate, resulting in an autophosphorylation event. This leads to the repression of the downstream negative regulator, BIN2, and induction of BR responses.
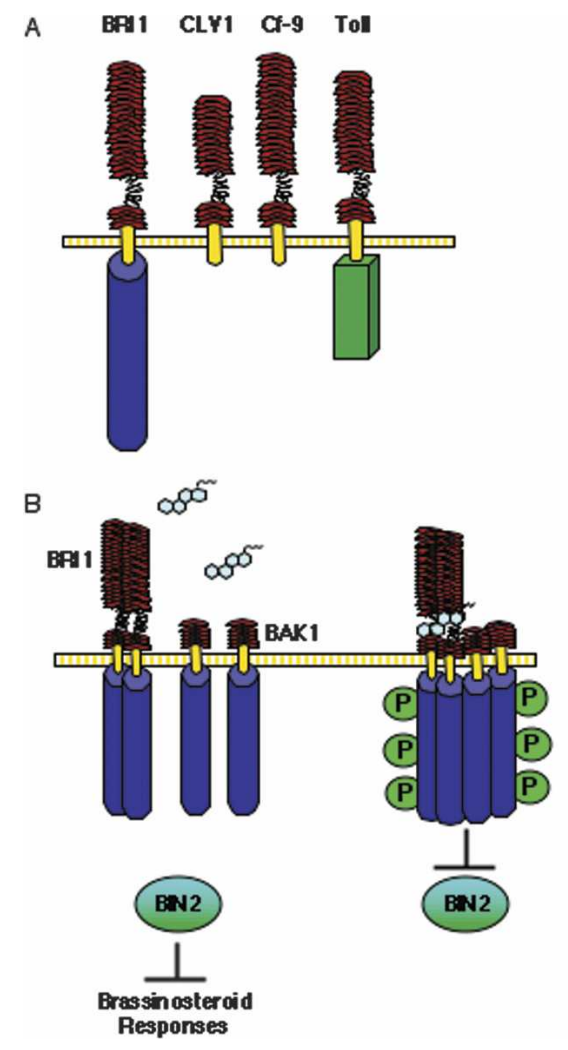

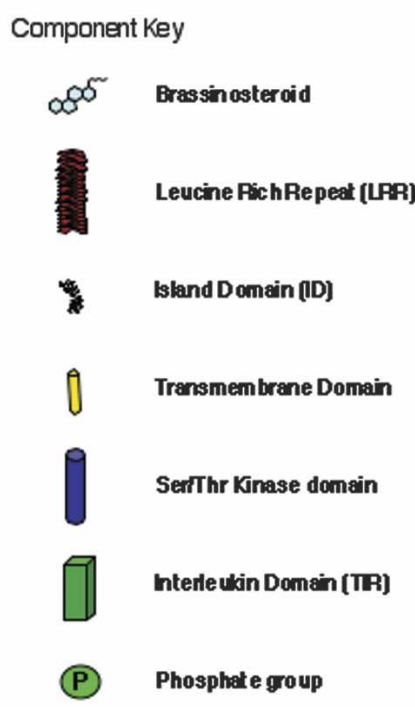

than directly through kinase domains. For example, the activated Toll receptor in Drosophila recruits, among other proteins, a serine/threonine kinase that shares similarity to the kinase domain found in plant LRRRLKs (Shiu and Bleecker 2001). Interestingly, other examples exist where plants have fused regulatory domains into a single protein while the equivalent domains in animals must associate through protein-protein interactions (Schena and Davis 1994).

The uncovering of a LRR-RLK as having a role in plant hormone signaling is perhaps not unexpected since this class of genes constitutes a large family consisting of $>200$ genes in the Arabidopsis genome (Shiu and Bleecker 2003). What was unexpected is that the extracellular LRR module directly binds BR. It is commonly thought that the LRR motif functions as a protein-protein interaction domain rather than as a domain that interacts with organic molecules (Kobe and Kajava 2001). In the case of the BRI1 LRR, however, the 25 leucine repeats are interrupted by a unique 70-amino-acid island domain (ID) between repeat 21 and 22 (Li and Chory 1997). The importance of the ID in BR signaling was implied by the observation that mutations that confer BR insensitivity cluster to this region and, more directly, by the ability of $\mathrm{BR}$ to bind to the ID in vitro (Li and Chory 1997; Kinoshita et al. 2005). Perhaps the ID explains the unique property of this extracellular domain to bind organic molecules, and it has been speculated that LRR22-ID may not fold in a typical way (Kinoshita et al. 2005).

In animals, a superfamily of nuclear receptors exists that are activated when the fully buried ligand completes the hydrophobic core of the receptor (Bogan et al. 1998). In this scenario, the ligand acts to stabilize the protein so that it can become nuclear localized and directly bind DNA. In plants, it appears that binding of BR to inactive BRI1 homodimers induces autophosphorylation of its cytoplasmic kinase domain and thereby stimulates the interaction of BRI1 with another related LRR-RLK called BAK1 (Fig. 4B). BAK1 contains a short four-leucine zipper repeat extracellular domain with no ID motif so it appears this LRR-RLK does not bind BR. Two models have been proposed for BRI1/BAK1 interactions. The first suggests that BR allows BRI1 and BAK1 to interact, thereby allowing transphosphorylation of BAK1. A second model is that BRI1 and BAK1 form a complex that is stabilized and activated by BR (Nam and Li 2002). Recently, in vitro analysis suggested that heteromeric associations between BRI1 and BAK1, as well as phosphorylation, are dependent on BR (Wang et al. 2005). Whatever the case, it appears that plants perceive steroid hormones at the plasma membrane and eventually stimulate gene expression through an involved signal transduction pathway that is similar to Wnt signaling in animals (Yin et al. 2002).

Binding experiments with BR indicate that it directly interacts with and activates the BRI1/BAK1 complex; however, some evidence exists to suggest that the first stages of signaling may be more complex. First, overexpressing the carboxypeptidase BRS1, which normally localizes to the extracellular space, can suppress a weak bri1 allele (Li et al. 2001a; Zhou and Li 2005). This suppression, which is specific to BRI1 and requires both BR 
and a functional protein kinase domain, indicates that BRS1 is involved in an early event in BRI1-mediated signaling. The molecular identity of BRS1 suggests that this protein may be involved in receptor proteolytic processing, and there is evidence that BRI1 may undergo endocytosis after binding BR (Russinova et al. 2004).

Alternatively, BRS1 may be involved in the processing of an as of yet unidentified protein ligand. The latter idea gains some support from studies in tomato showing that the BRI1 homolog is also the receptor for an 18-aminoacid protein called systemin (Montoya et al. 2002; Scheer et al. 2003). Systemin is the processed form of an inactive 200-amino-acid preprotein made during insect herbivory to activate a battery of defense genes (Ryan and Pearce 2003). Tomato bri1 mutants, which are phenotypically similar to BR-deficient dwarfs, are defective in systemin signaling. Furthermore, although BR does not reduce systemin binding, it does inhibit the systemin response (Scheer et al. 2003). It is possible that BR inhibits systemin signaling by directing the tomato BRI1 receptor away from systemin signaling and toward hormone signaling (Fig. 5A). Alternatively, BR and systemin may function through the same signaling pathway. In Arabidopsis, one function of a BR-bound BRI1 receptor is to negatively regulate the GSK3/SHAGGY-like kinase, BIN2 (Li et al. 2001b; Li and Nam 2002). In the absence of $\mathrm{BR}, \mathrm{BIN} 2$ negatively regulates activators of $\mathrm{BR}$ gene expression. In tomato, the BRI1 receptor is activated by systemin, which could influence BIN2 function. In this case, mutations in BIN2 and other downstream components of BR signaling may have altered systemin signaling. Although systemin has only been identified in some members of the Solanceae family, this observation of a shared receptor for both a peptide and organic molecule could mean that $\mathrm{BR}$ receptors in other plants may also have shared perception functions. Multiple recognition specificity may explain the discrepancy between the level of BRI protein, which is abundant in all tissues, and the low levels of BR ligand (Friedrichsen et al. 2000).

Aside from the structural similarities, other intriguing similarities exist between systemin/BR perception and signaling in tomato and Toll-based signaling in Drosophila. Like the tomato systemin/BR receptor, the Toll receptor also has two functions: one in embryogenesis, and the other in adult immune responses. Early events also appear to involve a proteolytic processing of an inactive preprotein to produce the activated Spatzle ligand that subsequently binds the extracellular LRR domain of the receptor (Jang et al. 2006). This leads to the recruitment of a serine/threonine kinase, which phosphorylates downstream components. In the case of the Toll pathway, however, a single ligand Spatzle is used in both processes but is cleaved by different developmentally regulated proteases into its mature form. The processing of the systemin ligand parallels that of the Toll ligand, as does the identification of a carboxyl peptidase in BRI1
A

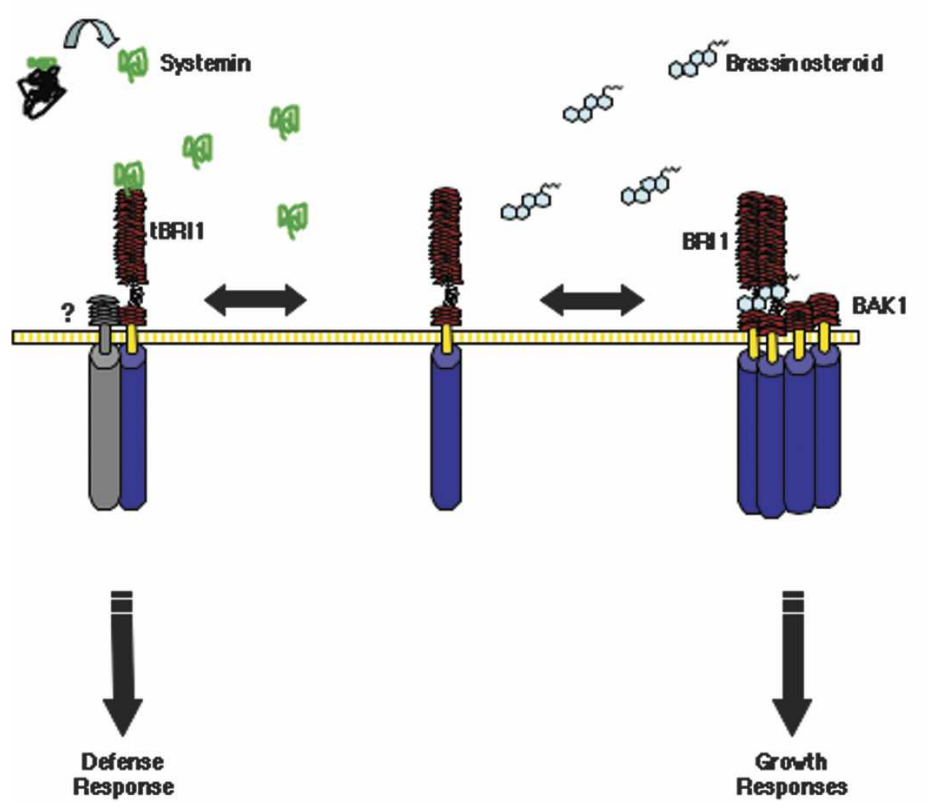

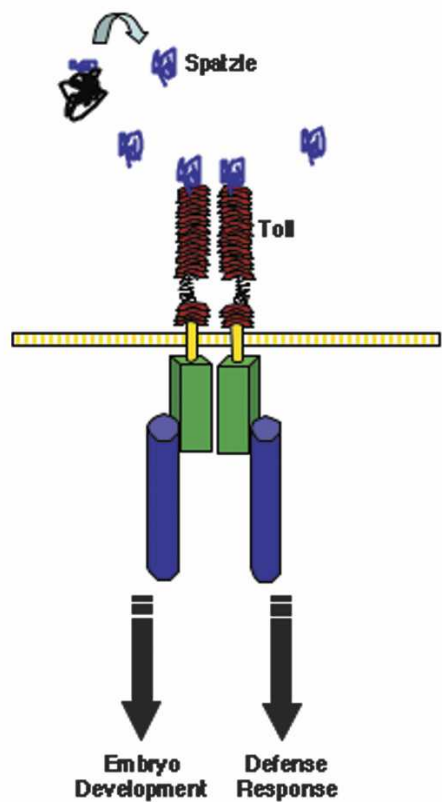

Figure 5. Consequences of LRR receptor-ligand interactions. (A) In tomato, the BRI1 receptor (tBRI1) can bind either BR or the small peptide systemin. Systemin is processed from a larger polypeptide by an as of yet uncharacterized protease (curved arrow). The resulting ligand binds tBRIl, causing heterodimerization of the tBRIl with an uncharacterized LRR protein (?) to induce defense responses. Alternatively, if tBRI1 binds BR, this results in a BRI-BAK1 dimerization and BR-dependent growth. (B) In Drosophila, a protease (curved arrow) cleaves a preprotein to generate a polypeptide Spatzle, which subsequently binds the Toll receptor. The choice between embryo development and innate immune response is determined by the developmentally regulated cleavage of the pre-Spatzle protein. 
signaling. Although not conserved at the amino acid level, the Toll receptor also contains an "island" within the LRR extracellular domains whose functions are unknown. Perhaps like BRI1, this region can also bind an organic molecule that modulates its function.

\section{Ubiquitination-based hormone receptors}

The first hints that some plant hormones had unique mechanisms of signaling came from the identification of genes required for normal responsiveness to exogenous auxin. Broadly speaking, these genes encoded two classes of molecules. One comprised members of a family of extremely shortlived nuclear proteins called Aux/IAAs (Rouse et al. 1998; Tian and Reed 1999; Nagpal et al. 2000; Rogg et al. 2001; for review, see Reed 2001). Aux/ IAA genes are rapidly induced by auxin application, and their protein products are then quickly degraded (Abel and Theologis 1996). Mutations in Aux/IAA genes that conferred increased resistance to auxin mapped to a common domain shown to be important in protein stability. Hence, decreasing the turnover of an Aux/IAA protein somehow conferred decreased auxin sensitivity. The sec- ond class of molecules identified various components of the SCF (Skp1/Cullin/F-box) ubiquitin protein complex (Ruegger et al. 1998; Gray et al. 1999, 2001).

The SCF complex, which was first identified in yeast, provides a mechanism by which cells can rapidly degrade proteins by targeting them to the $26 \mathrm{~S}$ proteosome (for review, see Tyers and Jorgensen 2000). Briefly, ubiquitin is first activated by an E1 enzyme and then passed to a family of ubiquitin-conjugating E2 enzymes (Fig. 6). The E2 enzyme carrying an ubiquitin molecule can interact with the Cullin subunit of the SCF complex. The SKP1 subunit acts as an adaptor protein between Cullin and the F-box so that when the F-box protein captures a protein target it is now in the vicinity of the E2 enzyme and can be ubiquinated (Fig. 6). This process is reiterated until the target protein accumulates more than four ubiquitins, which signal it for degradation via the $26 \mathrm{~S}$ proteosome.

There are other subunits and modifications that influence SCF function, but the specificity of the F-box protein in determining target substrate suggested that the SCF complex is central to the regulation of Aux/IAA protein levels. An F-box protein, TIR1, which specifi-
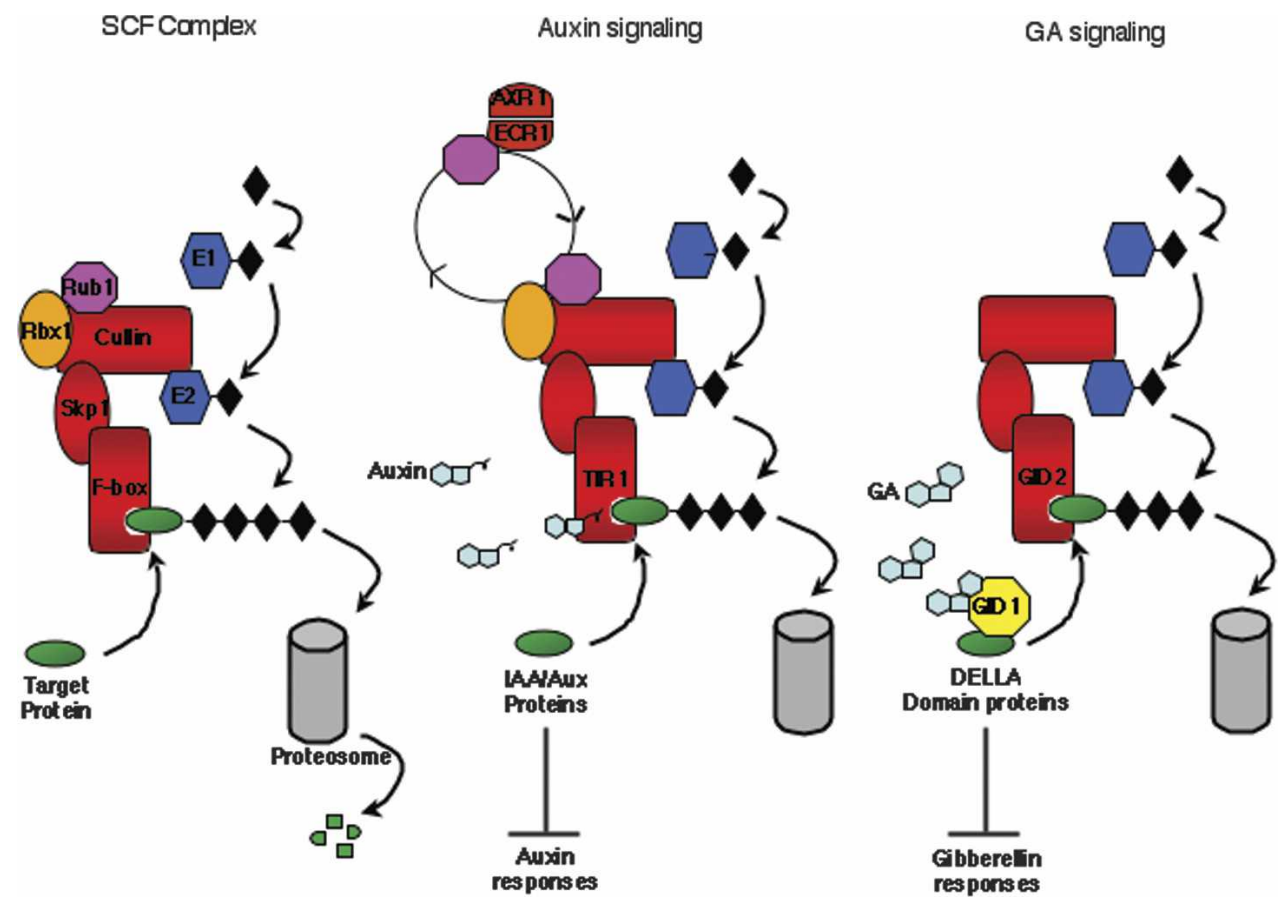

Figure 6. SCF-dependent hormone-regulated ubiquitination of proteins. Key components in a canonical SCF pathway are shown, and related components in the auxin and GA pathways have the same shape. The left panel represents a yeast-type SCF proteolysis model in which an ubiquitin ( ) is added to the target protein via a set of reactions. Ubiquitin is activated by an E1 enzyme and conjugated to the target via the E2 and F-box proteins associated with the SCF complex. The F-box protein determines specificity of targets. As ubiquitination of the target is a reiterative process, the ubiquitin tail grows and, after reaching a certain length, causes the degradation of the target via the 20S proteosome. The middle panel depicts auxin-mediated degradation of Aux/IAA repressor targets. The TIR 1 F-box protein binds auxin, and this stimulates its association with the Aux/IAA protein. Targeting of Aux/IAA to the proteosome releases auxin response genes from repression. The modification of the Cullin subunit with the ubiquitin-like protein RUB1 and other proteins enhances SCF-dependent ubiquitination. The first auxin resistant mutant (arx1) identified a protein required in rubinylation. The right panel depicts GA-mediated degradation of the DELLA domain repressor targets. The GID1 protein binds the DELLA protein in a GA-dependent manner and targets it to the SCF ${ }^{\mathrm{GID} 2}$ complex for ubiquitination. Degradation of the DELLA domain proteins releases GA-dependent genes from repression. 
cally targets the Aux/IAA proteins for proteolysis, was identified in a screen for resistance to auxin transport inhibitors, and subsequent analysis suggested it was an essential component of auxin responsiveness (Ruegger et al. 1998). The SCF ${ }^{\mathrm{TIR} 1}$ complex was shown to bind Aux/ IAA proteins directly, and this binding was enhanced in a cell free assay by the addition of auxin (Gray et al. 2001; Dharmasiri et al. 2003; Kepinski and Leyser 2004). Finally, it was shown that TIR1 itself could specifically bind bioactive auxin, and this resulted in an increased affinity for Aux/IAA proteins (Dharmasiri et al. 2005a; Kepinski and Leyser 2005). Thus TIR1 is an auxin receptor, and unlike most F-box substrate interactions, this F-box protein can be directly modified by a small organic molecule (Fig. 6).

Although Aux/IAA proteins are turned over in an auxin-dependent manner, these proteins do not bind DNA but heterodimerize with a related family of auxin response factor proteins (ARFs) that do bind DNA (Kim et al. 1997; Ulmasov et al. 1997). These findings led to a model suggesting that Aux/IAA proteins act as repressors of ARF-mediated transcription and that auxin activates the SCF ${ }^{\text {TIR } 1}$ complex to specifically degrade these repressors to allow ARF-dependent auxin responses. Strong support for this model came from knockout combinations of all four TIR1-related auxin receptor genes that not only caused severe loss of auxin responsiveness but also phenocopied many of the auxin related developmental defects seen in ARF loss-of-function mutants (Dharmasiri et al. 2005b).

The $\sim 700$ F-box proteins predicted to be encoded by the Arabidopsis genome is roughly one order of magnitude more than the number found in fungi and animals. This begs the question as to whether any other F-box proteins function as receptors for other plant hormones. One of the best candidates was an F-box protein involved in GA signaling. In both rice and Arabidopsis, loss-offunction mutations in GID2 and SLY1, respectively, result in reduced GA responsiveness, and both genes encode related F-box proteins (Steber et al. 1998; McGinnis et al. 2003; Sasaki et al. 2003). These F-box proteins appear to target a family of proteins that normally function to repress GA-related processes (Fig. 6; Peng et al. 1997; Silverstone et al. 1998; Dill et al. 2001; Itoh et al. 2002). Because the addition of GA causes these proteins to be degraded and thereby activates GA-dependent gene expression, the GA system has many similarities to the components involved in auxin signaling. This led to suggestions that perhaps GID2/SLY1 F-boxes could be GA receptors. However, a loss-of-function mutation in a rice gene encoding a protein with similarity to animal hormone lipases (GID1) was also identified, and this mutation caused both a reduction in GA responsiveness and prevented the degradation of the DELLA domain repressor protein SLR1 (Ueguchi-Tanaka et al. 2005). More importantly, GID1 specifically bound bioactive GA, and its ability to bind 17 SLR1 in a yeast two-hybrid assay was GA dependent (Ueguchi-Tanaka et al. 2005). Hence, the addition of GA results in a protein-protein interaction between GID1 and the SLR1 DELLA domain protein to cause the degradation of this negative regulator of the GA response (Fig. 6). The GID1 protein has no detectable lipase activity but is soluble and localizes to the nucleus where DELLA domain proteins reside. Interestingly, members of the TIR1 family of proteins also show nuclear localization, which raises questions of where these receptors encounter their hormones and whether the hormone influences receptor localization.

The identification of the SCF complexes and protein turnover as central to early auxin and GA signaling events suggests that the proteolysis of repressor proteins is an important hormone signal transduction mechanism in plants. However, unlike most epigenetic signaling events, such as a phosphotransfer, proteolysis is irreversible and therefore enforces directionality on a system. Such directionality is important in decisions that require total commitments such as controlling the cell cycle (Patton et al. 1998). By analogy, it could be argued that changes in levels of hormones such as auxin and GA during plant development should be followed by irreversible developmental commitments to processes such as cell division and expansion.

With respect to plant hormone signaling, the auxin and GA receptors are unique in that the number of components between the hormone ligand and the transcriptional factors they control is very small. Depending on the process being regulated, the length of the signaling pathway should reflect the required balance between the speed and robustness of a simple pathway versus the noise buffering ability of a more complex pathway. Buffering occurs because the addition of signaling components creates opportunities for loops to form that can enhance robustness while reducing signaling noise (Brandman et al. 2005). Hence, auxin and GA signaling should be very fast but with less buffering capacity than ethylene, BR, and cytokinin signaling. This modeling information should be useful when mapped onto the different physiological and developmental processes that these hormones influence.

\section{Hormone reception and plant development}

By definition, a hormone receptor should be able to recognize subtle structural differences between small molecules in a highly specific manner. Binding of the hormone to the receptor should be noncovalent and reversible and result in an activated complex that induces the primary hormone response. Certainly, the plant hormone receptors reviewed here fulfill many of these criteria, and in the cases of ethylene, cytokinin, and BR perception, the classic modules of receptor kinases have been identified. Having the receptors in hand has allowed comparison to each other as well as to those in other systems. For example, ethylene interferes with its two-component receptor function, whereas the cytokinin two-component receptor acts through the archetypal mechanism of activation and phosphotransfer to a response regulator. The ethylene two-component kinase does not signal to a canonical response regulator, and its negative regulation of receptor function suggests the re- 
ceptor has other functions in the absence of the hormone. In tomato, the BR receptor is not specific but is shared with another signaling pathway. Although the extracellular domain of BRI1 resembles the classic LRRprotein interaction motif, it has the capacity to bind an organic molecule through a novel embedded domain. Presumably the extracellular LRR originally served as a protein ligand receptor and the addition of the BR-binding ID domain arose secondarily.

These observations suggest that hormone receptors like those involving ethylene and BR signaling have additional functions that the hormones influence. Auxin and GA perception use a unique SCF-based proteolysis mechanism that takes advantage of F-box specificity for target proteins. However, in the case of GA-dependent targets, different hormones and environmental stresses such as salt can also influence target stability (Achard et al. 2003, 2006; Fu and Harberd 2003). The use of SCFbased proteolysis as a mechanism of signaling creates potential nodes of interactions between signaling molecules. For example, specific mutations that conferred auxin insensitivity and mapped to a Cullin subunit also altered jasmonate sensitivity by attenuating the assembly of the jasmonate specific F-box protein COI1 into the SCF complex (Ren et al. 2005).

These molecular mechanisms of hormone perception are now beginning to explain how these small enigmatic molecules can coordinate plant growth and development. Plant hormones have never had a clear location of synthesis and appear to cause a myriad of responses. For these reasons the role of hormones as "limiting factors" whose levels control a particular developmental pathway has never resonated well and has led to arguments that these small molecules actually function more as cofactors in balancing the different reactions and metabolic pathways comprising the overall network driving development (Trewavas 1986). The non-Euclidian patterns of connectivity in a network not only buffer a system but also lead to the capacity to produce the same output via different strategies, which fits well with plant hormone phenomena. In this respect, that the loss of hormone perception does not generally result in overall lethality or even a reduction of cell types also supports the notion that hormones modulate rather than limit particular processes. Being perceived by proteins that have additional functions, or acting at central nodes of signaling, partly supports a modulating versus control and command scenario for plant hormone function.

Whatever the case, it is clear there are differences between how plants and animals use organic-based hormones to organize tissues and organs. Unlike animals, plants are essentially an amalgamation of loosely dependent units that do not require highly centralized centers to coordinate growth and development. Because of this, different parts of a plant can experience different local environments and respond somewhat independently. Consequently, rather than having specific organs that make and respond to particular hormones, each independent unit produces and responds to many signals. By acting as factors that bind with high specificity to proteins with additional roles in development, plant hormones can add flexibility to signaling pathways that underlie the plasticity of development seen in higher plants.

\section{Acknowledgments}

P.M. is a participant in the Collaborative Program in Developmental Biology.

\section{References}

Abel, S. and Theologis, A. 1996. Early genes and auxin action. Plant Physiol. 111: 9-17.

Achard, P., Vriezen, W.H., van Der Straeten, D., and Harberd, N.P. 2003. Ethylene regulates Arabidopsis development via the modulation of DELLA protein growth repressor function. Plant Cell 15: 2816-2825.

Achard, P., Cheng, H., De Grauwe, L., Decat, J., Schoutteten, H., Moritz, T., Van Der Straeten, D., Peng, J., and Harberd, N.P. 2006. Integration of plant responses to environmentally activated phytohormonal signals. Science 311: 91-94.

Bleecker, A., Estelle, M., Somerville, C., and Kende, H. 1988. Insensitivity to ethylene conferred by a dominant mutation in Arabidopsis thaliana. Science 241: 1086-1089.

Bogan, A.A., Cohen, F.E., and Scanlan, T.S. 1998. Natural ligands of nuclear receptors have conserved volumes. Nat. Struct. Biol. 5: 679-681.

Brandman, O., Ferrell, J.E., Li, R., and Meyer, T. 2005. Interlinked fast and slow positive feedback loops drive reliable cell decisions. Science 310: 496-498.

Cancel, J.D. and Larsen, P.B. 2002. Loss-of-function mutations in the ethylene receptor ETR1 cause enhanced sensitivity and exaggerated response to ethylene in Arabidopsis. Plant Physiol. 129: 1557-1567.

Chang, C., Kwok, S.F., Bleecker, A.B., and Meyerowitz, E.M. 1993. Arabidopsis ethylene response gene ETR1: Similarity of product to two-component regulators. Science 262: 539544.

Chen, Y.F., Randlett, M.D., Findell, J.L., and Schaller, G.E. 2002. Localization of the ethylene receptor ETR1 to the endoplasmic reticulum of Arabidopsis. J. Biol. Chem. 277: 19861-19866.

Chen, Y.F., Etheridge, N., and Schaller, G.E. 2005. Ethylene signal transduction. Ann. Bot. (Lond.) 95: 901-915.

Clark, K.L., Larsen, P.B., Wang, X., and Chang, C. 1998. Association of the Arabidopsis CTR1 Raf-like kinase with the ETR1 and ERS1 ethylene receptors. Proc. Nat1. Acad. Sci. 95: 5401-5406.

Clouse, S.D., Langford, M., and McMorris, T.C. 1996. A brassinosteroid-insensitive mutant in Arabidopsis thaliana exhibits multiple defects in growth and development. Plant Physiol. 111: 671-678.

Davies, P.J. 1995. The plant hormones: Their nature, occurrence and functions. In Plant hormones: Physiology, biochemistry and molecular biology (ed. P.J. Davies), pp. 1-12. Kluwer Academic Publishers, Dordrecht, The Netherlands.

Dharmasiri, N., Dharmasiri, S., Jones, A.M., and Estelle, M. 2003. Auxin action in a cell-free system. Curr. Biol. 13: 1418-1422.

Dharmasiri, N., Dharmasiri, S., and Estelle, M. 2005a. The Fbox protein TIR1 is an auxin receptor. Nature 435: 441-445.

Dharmasiri, N., Dharmasiri, S., Weijers, D., Lechner, E., Yamada, M., Hobbie, L., Ehrismann, J.S., Jurgens, G., and Estelle, M. 2005b. Plant development is regulated by a family 
of auxin receptor F box proteins. Dev. Cell 9: 109-119.

Dill, A., Jung, H.S., and Sun, T.-P. 2001. The DELLA motif is essential for gibberellin induced degradation of RGA. Proc. Natl. Acad. Sci. 98: 14162-14167.

Friedrichsen, D.M., Joazeiro, C.A., Li, J., Hunter, T., and Chory, J. 2000. Brassinosteroid-insensitive- 1 is a ubiquitously expressed leucine-rich repeat receptor serine/threonine kinase. Plant Physiol. 123: 1247-1256.

Fu, X. and Harberd, N.P. 2003. Auxin promotes Arabidopsis root growth by modulating gibberellin response. Nature 421: 740-743.

Gao, Z., Chen, Y.F., Randlett, M.D., Zhao, X.C., Findell, J.L., Kieber, J.J., and Schaller, G.E. 2003. Localization of the Raflike kinase CTR1 to the endoplasmic reticulum of Arabidopsis through participation in ethylene receptor signalling complexes. J. Biol. Chem. 278: 34725-34732.

Gray, W.M., del Pozo, J.C., Walker, L., Hobbie, L., Risseeuw, E., Banks, T., Crosby, W.L., Yang, M., Ma, H., and Estelle, M. 1999. Identification of an SCF ubiquitin-ligase complex required for auxin response in Arabidopsis thaliana. Genes \& Dev. 13: 1678-1691.

Gray, W.M., Kepinski, S., Rouse, D., Leyser, O., and Estelle, M. 2001. Auxin regulates SCF(TIR1)-dependent degradation of AUX/IAA proteins. Nature 414: 271-276.

Higuchi, M., Pischke, M.S., Mahonen, A.P., Miyawaki, K., Hashimoto, Y., Seki, M., Kobayashi, M., Shinozaki, K., Kato, T., Tabata, S., et al. 2004. In planta functions of the Arabidopsis cytokinin receptor family. Proc. Nat1. Acad. Sci. 101: $8821-8826$.

Hua, J. and Meyerowitz, E.M. 1998. Ethylene responses are negatively regulated by a receptor gene family in Arabidopsis thaliana. Cell 94: 261-271.

Hua, J., Chang, C., Sun, Q., and Meyerowitz, E.M. 1995. Ethylene insensitivity conferred by Arabidopsis ERS gene. Science 269: 1712-1714.

Huang, Y., Li, H., Hutchison, C.E., Laskey, J., and Kieber, J.J. 2003. Biochemical and functional analysis of CTR1, a protein kinase that negatively regulates ethylene signaling in Arabidopsis. Plant J. 33: 221-233.

Hwang, I. and Sheen, J. 2001. Two-component circuitry in Arabidopsis cytokinin signal transduction. Nature 413: 383-389.

Inoue, T., Higuchi, M., Hashimoto, Y., Seki, M., Kobayashi, M., Kato, T., Tabata, S., Shinozaki, K., and Kakimoto, T. 2001. Identification of CRE1 as a cytokinin receptor from Arabidopsis. Nature 409: 1060-1063.

Itoh, H., Ueguchi-Tanaka, M., Sato, Y., Ashikari, M., and Matsuoka, M. 2002. The gibberellin signaling pathway is regulated by the appearance and disappearance of SLENDER RICE1 in nuclei. Plant Cell 14: 57-70.

Jang, I.H., Chosa, N., Kim, S.H., Nam, H.J., Lemaitre, B., Ochiai, M., Kambris, Z., Brun, S., Hashimoto, C., Ashida, M., et al. 2006. A Spatzle-processing enzyme required for Toll signaling activation in Drosophila innate immunity. Dev. Cell 10: 45-55.

Kato, M., Mizuno, T., Shimizu, T., and Hakoshima, T. 1997. Insights into multistep phosphorelay from the crystal structure of the C-terminal HPt domain of ArcB. Cell 88: 717723.

Kepinski, S. and Leyser, O. 2004. Auxin-induced SCFTIR1-Aux/ IAA interaction involves stable modification of the SCFTIR1 complex. Proc. Natl. Acad. Sci. 101: 12381-12386.

- 2005. The Arabidopsis F-box protein TIR 1 is an auxin receptor. Nature 435: 446-451.

Kieber, J.J., Rothenberg, M., Roman, G., Feldman, K.A., and Ecker, J.R. 1993. CTR1, a negative regulator of the ethylene response pathway in Arabidopsis, encodes a member of the Raf family of protein kinases. Cell 72: 427-441.

Kim, J., Harter, K., and Theologis, A. 1997. Protein-protein interactions among the Aux/IAA proteins. Proc. Natl. Acad. Sci. 94: 11786-11791.

Kinoshita, T., Cano-Delgado, A., Seto, H., Hiranuma, S., Fujioka, S., Yoshida, S., and Chory, J. 2005. Binding of brassinosteroids to the extracellular domain of plant receptor kinase BRI1. Nature 433: 167-171.

Kobe, B. and Kajava, A.V. 2001. The leucine-rich repeat as a protein recognition motif. Curr. Opin. Struct. Biol. 11: 725732.

Li, J. and Chory, J. 1997. A putative leucine-rich repeat receptor kinase involved in brassinosteroid signal transduction. Cell 90: 929-938.

Li, J. and Nam, K.H. 2002. Regulation of brassinosteroid signaling by a GSK3/SHAGGY-like kinase. Science 295: 12991301.

Li, J., Nagpal, P., Vitart, V., McMorris, T.C., and Chory, J. 1996. A role for brassinosteroids in light-dependent development of Arabidopsis. Science 272: 398-401.

Li, J., Lease, K.A., Tax, F.E., and Walker, J.C. 2001a. BRS1, a serine carboxypeptidase, regulates BRIl signaling in Arabidopsis thaliana. Proc. Natl. Acad. Sci. 98: 5916-5921.

Li, J., Nam, K.H., Vafeados, D., and Chory, J. 2001b. BIN2, a new brassinosteroid-insensitive locus in Arabidopsis. Plant Physiol. 127: 14-22.

McGinnis, K.M., Thomas, S.G., Soule, J.D., Strader, L.C., Zale, J.M., Sun, T.-P., and Steber, C.M. 2003. The Arabidopsis SLEEPY1 gene encodes a putative F-box subunit of an SCF E3 ubiquitin ligase. Plant Cell 15: 1120-1130.

Mizuno, T. 1997. Compilation of all genes encoding two-component phosphotransfer signal transducers in the genome of Escherichia coli. DNA Res. 4: 161-168.

Montoya, T., Nomura, T., Farrar, K., Kaneta, T., Yokota, T., and Bishop, G.J. 2002. Cloning the tomato curl3 gene highlights the putative dual role of the leucine-rich repeat receptor kinase tBRI1/SR160 in plant steroid hormone and peptide hormone signaling. Plant Cell 14: 3163-3176.

Nagpal, P., Walker, L.M., Young, J.C., Sonawala, A., Timpte, C., Estelle, M., and Reed, J.W. 2000. AXR2 encodes a member of the Aux/IAA protein family. Plant Physiol. 123: 563-574.

Nam, K.H. and Li, J. 2002. BRI1/BAK1, a receptor kinase pair mediating brassinosteroid signaling. Cell 110: 203-212.

Noguchi, T., Fujioka, S., Choe, S., Takatsuto, S., Yoshida, S., Yuan, H., Feldmann, K.A., and Tax, F.E. 1999. Brassinosteroid-insensitive dwarf mutants of Arabidopsis accumulate brassinosteroids. Plant Physiol. 121: 743-752.

O'Malley, R.C., Rodriguez, F.I., Esch, J.J., Binder, B.M., O'Donnell, P., Klee, H.J., and Bleecker, A.B. 2005. Ethylenebinding activity, gene expression levels, and receptor system output for ethylene receptor family members from Arabidopsis and tomato. Plant J. 41: 651-659.

Patton, E.E., Willems, A.R., and Tyers, M. 1998. Combinatorial control in ubiquitin-dependent proteolysis: Don't Skp the F-box hypothesis. Trends Genet. 14: 236-243.

Peng, J., Carol, P., Richards, D.E., King, K.E., Cowling, R.J., Murphy, G.P., and Harberd, N.P. 1997. The Arabidopsis GAI gene defines a signaling pathway that negatively regulates gibberellin responses. Genes \& Dev. 11: 3194-3205.

Qu, X. and Schaller, G.E. 2004. Requirement of the histidine kinase domain for signal transduction by the ethylene receptor ETR1. Plant Physiol. 136: 2961-2970.

Razem, F.A., El-Kereamy, A., Abrams, S.R., and Hill, R.D. 2006. The RNA-binding protein FCA is an abscisic acid receptor. Nature 439: 290-294. 
Reed, J. 2001. Roles and activities of Aux/IAA proteins in Arabidopsis. Trends Plant Sci. 6: 420-425.

Ren, C., Pan, J., Peng, W., Genschik, P., Hobbie, L., Hellmann, H., Estelle, M., Gao, B., Peng, J., Sun, C., et al. 2005. Point mutations in Arabidopsis Cullin1 reveal its essential role in jasmonate response. Plant J. 42: 514-524.

Rodriguez, F.I., Esch, J.J., Hall, A.E., Binder, B.M., Schaller, G.E., and Bleecker, A.B. 1999. A copper cofactor for the ethylene receptor ETR1 from Arabidopsis. Science 283: 996-998.

Rogg, L.E., Lasswell, J., and Bartel, B. 2001. A gain-of-function mutation in IAA28 suppresses lateral root development. Plant Cell 13: 465-480.

Rouse, D., Mackay, P., Stirnberg, P., Estelle, M., and Leyser, O. 1998. Changes in auxin response from mutations in an AUX/ IAA gene. Science 279: 1371-1373.

Ruegger, M., Dewey, E., Gray, W.M., Hobbie, L., Turner, J., and Estelle, M. 1998. The TIR1 protein of Arabidopsis functions in auxin response and is related to human SKP2 and yeast Grrlp. Genes \& Dev. 12: 198-207.

Russinova, E., Borst, J.W., Kwaaitaal, M., Cano-Delgado, A., Yin, Y., Chory, J., and de Vries, S.C. 2004. Heterodimerization and endocytosis of Arabidopsis brassinosteroid receptors BRI1 and AtSERK3 (BAK1). Plant Cell 16: 3216-3229.

Ryan, C.A. and Pearce, G. 2003. Systemins: A functionally defined family of peptide signals that regulate defensive genes in Solanaceae species. Proc. Natl. Acad. Sci. (Suppl. 2) 100: 14577-14580.

Sakai, H., Hua, J., Chen, Q.G., Chang, C., Medrano, L.J., Bleecker, A.B., and Meyerowitz, E.M. 1998. ETR2 is an ETR1-like gene involved in ethylene signaling in Arabidopsis. Proc. Natl. Acad. Sci. 95: 5812-5817.

Sasaki, A., Itoh, H., Gomi, K., Ueguchi-Tanaka, M., Ishiyama, K., Kobayashi, M., Jeong, D.H., An, G., Kitano, H., Ashikari, M., et al. 2003. Accumulation of phosphorylated repressor for gibberellin signaling in an F-box mutant. Science 299: 1896-1898.

Schaller, G.E. and Bleecker, A.B. 1995. Ethylene-binding sites generated in yeast expressing the Arabidopsis ETR1 gene. Science 270: 1809-1811.

Scheer, J.M., Pearce, G., and Ryan, C.A. 2003. Generation of systemin signaling in tobacco by transformation with the tomato systemin receptor kinase gene. Proc. Natl. Acad. Sci. 100: 10114-10117.

Schena, M. and Davis, R.W. 1994. Structure of homeoboxleucine zipper genes suggests a model for the evolution of gene families. Proc. Natl. Acad. Sci. 91: 8393-8397.

Shiu, S.H. and Bleecker, A.B. 2001. Receptor-like kinases from Arabidopsis form a monophyletic gene family related to animal receptor kinases. Proc. Natl. Acad. Sci. 98: 10763-10768.

- 2003. Expansion of the receptor-like kinase/Pelle gene family and receptor-like proteins in Arabidopsis. Plant Physiol. 132: 530-543.

Silverstone, A.L., Ciampaglio, C.N., and Sun, T. 1998. The Arabidopsis RGA gene encodes a transcriptional regulator repressing the gibberellin signal transduction pathway. Plant Cell 10: 155-169.

Steber, C.M., Cooney, S.E., and McCourt, P. 1998. Isolation of the GA-response mutant sly1 as a suppressor of ABI1-1 in Arabidopsis thaliana. Genetics 149: 509-521.

Suzuki, T., Miwa, K., Ishikawa, K., Yamada, H., Aiba, H., and Mizuno, T. 2001. The Arabidopsis sensor His-kinase, AHk4, can respond to cytokinins. Plant Cell Physiol. 42: 107-113.

Szekeres, M., Nemeth, K., Koncz-Kalman, Z., Mathur, J., Kauschmann, A., Altmann, T., Redei, G.P., Nagy, F., Schell, J., and Koncz, C. 1996. Brassinosteroids rescue the deficiency of
CYP90, a cytochrome P450, controlling cell elongation and de-etiolation in Arabidopsis. Cell 85: 171-182.

Tian, Q. and Reed, J.W. 1999. Control of auxin-regulated root development by the Arabidopsis thaliana SHY2/IAA3 gene. Development 126: 711-721.

Trewavas, A. 1986. Understanding the control of plant development and the role of growth substances. Aust. I. Plant Physiol. 13: 447-457.

Tyers, M. and Jorgensen, P. 2000. Proteolysis and the cell cycle: With this RING I do thee destroy. Curr. Opin. Genet. Dev. 10: 54-64.

Ueguchi-Tanaka, M., Ashikari, M., Nakajima, M., Itoh, H., Katoh, E., Kobayashi, M., Chow, T.Y., Hsing, Y.I., Kitano, H., Yamaguchi, I., et al. 2005. GIBBERELLIN INSENSITIVE DWARF1 encodes a soluble receptor for gibberellin. Nature 437: 693-698.

Ulmasov, T., Murfett, J., Hagen, G., and Guilfoyle, T.J. 1997. Aux/IAA proteins repress expression of reporter genes containing natural and highly active synthetic auxin response elements. Plant Cell 9: 1963-1971.

Wang, X., Goshe, M.B., Soderblom, E.J., Phinney, B.S., Kuchar, J.A., Li, J., Asami, T., Yoshida, S., Huber, S.C., and Clouse, S.D. 2005. Identification and functional analysis of in vivo phosphorylation sites of the Arabidopsis BRASSINOSTEROID-INSENSITIVE1 receptor kinase. Plant Cell 17: 1685-1703.

Wilkins, A.S. 1995. Moving up the hierarchy: A hypothesis on the evolution of a genetic sex determination pathway. Bioessays 17: 71-77.

Yamada, H., Suzuki, T., Terada, K., Takei, K., Ishikawa, K., Miwa, K., Yamashino, T., Mizuno, T. 2001. The Arabidopsis AHK4 histidine kinase is a cytokinin binding receptor that transduces cytokinin signals across the membrane. Plant Cell Physiol. 42: 1017-1023.

Yanagisawa, S., Yoo, S.D., and Sheen, J. 2003. Differential regulation of EIN3 stability by glucose and ethylene signalling in plants. Nature 425: 521-525.

Yin, Y., Wang, Z.Y., Mora-Garcia, S., Li, J., Yoshida, S., Asami, T., and Chory, J. 2002. BES1 accumulates in the nucleus in response to brassinosteroids to regulate gene expression and promote stem elongation. Cell 109: 181-191.

Zhou, A. and Li, J. 2005. Arabidopsis BRS1 is a secreted and active serine carboxypeptidase. J. Biol. Chem. 280: 35554 35561. 


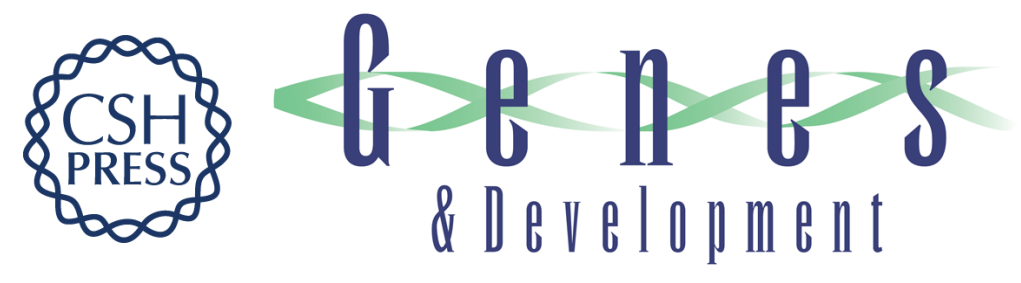

\section{Plant hormone receptors: perception is everything}

Brenda Chow and Peter McCourt

Genes Dev. 2006, 20:

Access the most recent version at doi:10.1101/gad.1432806

References This article cites 82 articles, 47 of which can be accessed free at: http://genesdev.cshlp.org/content/20/15/1998.full.html\#ref-list-1

License

Email Alerting Receive free email alerts when new articles cite this article - sign up in the box at the top Service right corner of the article or click here.

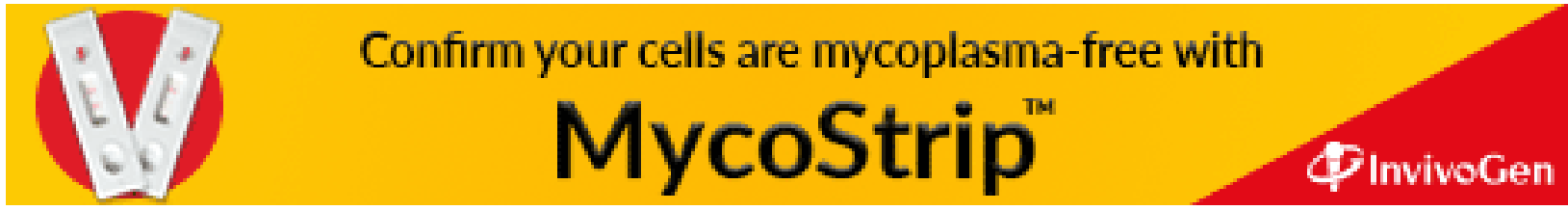

